\title{
Homodyne Tomography of a Single Photon Retrieved on Demand from a Cavity-Enhanced Cold Atom Memory
}

\author{
Erwan Bimbard, Rajiv Boddeda, Nicolas Vitrant, Andrey Grankin, Valentina Parigi, \\ Jovica Stanojevic, Alexei Ourjoumtsev, and Philippe Grangier \\ Laboratoire Charles Fabry, Institut d'Optique, CNRS, Univ. Paris Sud, 2 avenue Augustin Fresnel, 91127 Palaiseau cedex, France
}

(Received 4 October 2013; published 21 January 2014)

\begin{abstract}
We experimentally demonstrate that a nonclassical state prepared in an atomic memory can be efficiently transferred to a single mode of free-propagating light. By retrieving on demand a single excitation from a cold atomic gas, we realize an efficient source of single photons prepared in a pure, fully controlled quantum state. We characterize this source using two detection methods, one based on photon-counting analysis and the second using homodyne tomography to reconstruct the density matrix and Wigner function of the state. The latter technique allows us to completely determine the mode of the retrieved photon in its fine phase and amplitude details and demonstrate its nonclassical field statistics by observing a negative Wigner function. We measure a photon retrieval efficiency up to $82 \%$ and an atomic memory coherence time of $900 \mathrm{~ns}$. This setup is very well suited to study interactions between atomic excitations and use them in order to create and manipulate more sophisticated quantum states of light with a high degree of experimental control.
\end{abstract}

PACS numbers: 42.50.Dv, 03.67.-a, 37.10.-x, 42.50.Nn

Precisely controlling quantum states of light is crucial for many quantum information processing (QIP) tasks. In this respect, cold atomic ensembles are a very versatile tool. In the past decade, they have been extensively used to store and retrieve quantum states of light [1] and, more recently, to manipulate them using atomic interactions [2-4]. Quite often, however, the resulting quantum states were characterized only partially, typically by photon correlation measurements insensitive to the exact frequency or temporal envelope of the emitted photons. Although independent atomic ensembles have been shown to emit photons with a good degree of indistinguishability [5-8], their exact wave functions remained to be characterized. Demonstrating that strongly nonclassical states stored in an atomic ensemble can be retrieved on demand as single-mode, Fouriertransform limited light pulses is an important requirement to use such systems in QIP and to connect them to other QIP devices.

A very convenient way to fully characterize the retrieved quantum state is to use homodyne tomography [9], by reconstructing the photons' density matrix and Wigner function from the quadrature distributions of the light field measured by interference with a bright local oscillator. Since this technique characterizes the light's field rather than its intensity, it is intrinsically single mode and very sensitive to optical losses. Therefore, observing nonclassical features in the reconstructed quantum state proves that it can truly be emitted on demand, detected with a high efficiency, and controlled in all of its degrees of freedom which must exactly match those of the local oscillator. For a nonclassical state, the field's quadrature statistics are described by a non-Gaussian Wigner function taking negative values: losses or imperfections in the spatial mode, the polarization, the temporal envelope, or the frequency of the state will degrade the signal and eventually make its Wigner function completely positive. This makes homodyne characterization more precise but also technically more challenging than photon correlation measurements, which can be made insensitive to many of these imperfections. Ubiquitous in the characterization of Gaussian continuous-variable quantum memories [10-14], this technique has been extended during the past decade to analyze non-Gaussian states produced with heralded parametric or hot atomic vapor light sources $[9,15]$, and very recently with an optical quantum memory [16]. Transposing it to a cold atomic ensemble, where quantum light states can not only be stored but also processed, should form an ideal system for studying nonlinear transformations of few-photon states due to atomic interactions.

In this Letter we describe an experimental homodyne tomography of a single photon retrieved on demand from a cloud of cold rubidium atoms placed within an optical cavity. We show that both our atomic system and our detection setup are sufficiently efficient to preserve the negativity of the Wigner function directly reconstructed from raw experimental data. Taking into account the imperfections of the homodyne detector allows us to characterize the atomic system alone and to show that it can operate as a quantum memory with a 900 ns storage time, emitting free-propagating single photons in a well-controlled single spatiotemporal mode with an efficiency up to $82 \%$.

Our single-photon source is based on the Duan-LukinCirac-Zoller protocol [17], implemented in a cloud of cold ${ }^{87} \mathrm{Rb}$ atoms (Fig. 1), in a pulsed scheme. In the weak 


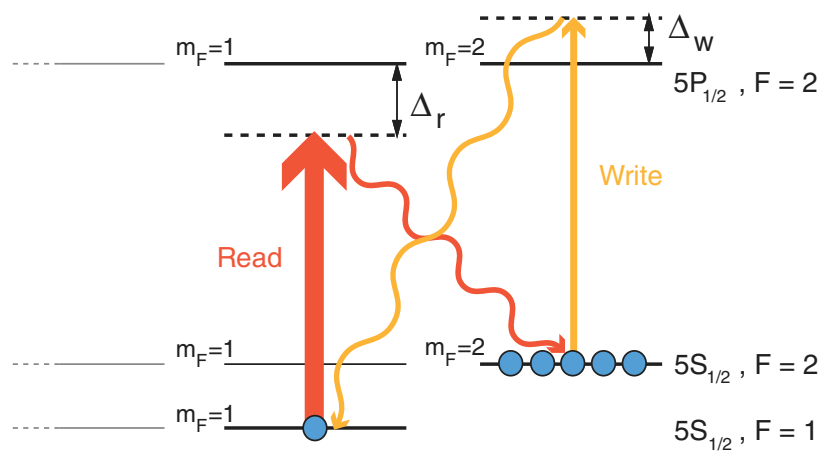

FIG. 1 (color online). Duan-Lukin-Cirac-Zoller protocol. In a cloud of ${ }^{87} \mathrm{Rb}$ atoms, two Raman transitions are used to store (write) and retrieve (read) a single photon as a collective excitation. Series of consecutive pulses of write and read beams are sent, with detunings $\Delta_{W}$ and $\Delta_{R}$, respectively.

driving limit, detecting a spontaneous Raman "write" photon heralds the creation of a single collective atomic spin excitation, which can be stored for a variable time and retrieved as a single "read" photon by another Raman step. The collective, phase-matched character of the four-photon process greatly enhances the photon retrieval efficiency in the observed mode, further improved by placing the atoms in a low-finesse optical cavity resonant with the emitted photons [18]. The maximal theoretical retrieval efficiency $\eta_{\max }=C /(1+C)$ depends only on the cooperativity $C=$ $N g^{2} / \kappa \gamma$ of the $N$ atoms coupled to the cavity field with a single-atom vacuum Rabi frequency $g$, with $\gamma$ and $\kappa$ being, respectively, the atomic dipole and the cavity field decay rates. In order to retrieve a Fourier-limited single-photon pulse with a constant phase profile, the frequency and time dependence of the read laser pulse are adjusted according to Ref. [19]. In practice, the retrieval efficiency is also reduced by various defects and decoherence sources.

The experimental setup is sketched in Fig. 2. A magnetooptically trapped cloud of ${ }^{87} \mathrm{Rb}$ is polarization-gradient cooled to $\approx 50 \mu \mathrm{K}$ and released in free fall inside a $66 \mathrm{~mm}$ long vertical Fabry-Perot cavity with a finesse $F=120$ and a linewidth $\kappa / 2 \pi=10 \mathrm{MHz}$. The cavity sustains a fundamental mode of waist $w_{0}=86 \mu \mathrm{m}$ at $795 \mathrm{~nm}$, coupled to $\sim 10^{4}$ atoms, providing the system with a cooperativity $C \approx 15$ on the $D 1$ transition. The difference in the cavity mirrors' reflectivities $\left(R^{\text {top }}=99.99 \%\right.$, $R^{\text {bottom }}=95 \%$ ) ensures that the photons emitted in this mode escape the cavity through the bottom mirror with a nearly $100 \%$ probability.

After an initial $100 \mu$ s step of optical pumping to the state $5 S_{1 / 2}, F=2, m_{F}=+2$, the atoms are excited with a series of counterpropagating write and read laser pulses, alternating with a $1.5 \mu$ s period during $1 \mathrm{~ms}$, before optical pumping quality degrades. This pair of pumping-measurement steps is then repeated 12 times before the atoms are recaptured and recooled for $120 \mathrm{~ms}$ and the sequence starts again, allowing us to perform $\approx 60000$ write-read trials

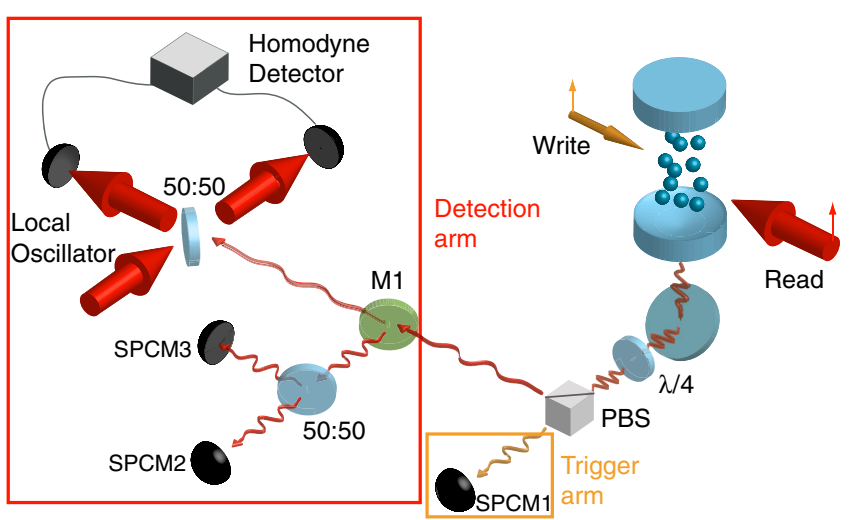

FIG. 2 (color online). Experimental setup. A cloud of cold rubidium atoms trapped inside an optical cavity is off-resonantly excited with alternating write and read pulses, creating pairs of photons Raman scattered in the cavity. They are split according to their polarization on a polarizing beam splitter (PBS). The detection of a write photon by the single-photon counting module SPCM 1 heralds the creation of a single atomic spin excitation, retrieved by the read pulse as a single photon directed either to SPCMs 2 and 3 for photon-counting measurements or to a homodyne detection setup. Mirror M1 is flipped in or out according to the choice of detector.

per second. The write and read pulses are both $\pi$ polarized and propagate orthogonally to the quantization axis, oriented along the cavity. Their temporal intensity profiles are nearly Gaussian, with a $1 / e$ half-width $\sigma_{w}=\sigma_{r}=$ $80 \mathrm{~ns}$ and an adjustable delay. The write pulse is detuned by $\Delta_{W} / 2 \pi=+28 \mathrm{MHz}$ from the $5 S_{1 / 2}, \quad F=2$, $m_{F}=2 \rightarrow 5 P_{1 / 2}, F=2, m_{F}=2$ transition, making the write photon emitted by Raman scattering towards the $5 S_{1 / 2}, \quad F=1, m_{F}=1$ state resonant with a $\mathrm{TEM}_{00}$ cavity mode. Similarly, after accounting for a linear resonance shift induced by the atoms, a read pulse detuning of $\Delta_{R} / 2 \pi=-46 \mathrm{MHz}$ from the $5 S_{1 / 2}, F=1$, $m_{F}=1 \rightarrow 5 P_{1 / 2}, F=1, m_{F}=1$ makes the emitted read Raman photon bringing the system back to the initial $5 S_{1 / 2}$, $F=2, m_{F}=+2$ state resonant with another $\mathrm{TEM}_{00}$ cavity mode, shifted from the previous one by 3 cavity free spectral ranges. After escaping the cavity, the photons are separated according to their opposite circular polarizations. The write photons are then spatially and spectrally filtered via a single-mode optical fiber, interference filters, and a Fabry-Perot cavity, designed to block stray light from other beams, before being detected with a single-photon counting module SPCM 1. With a maximal Rabi frequency $\Omega_{W}^{\max } / 2 \pi \approx 200 \mathrm{kHz}$ for the write pulse, and taking into account the $33 \%$ total trigger path detection efficiency, a write photon is detected on SPCM 1 with a probability $\approx 10^{-3}$ per write pulse, which makes the probability to create multiple excitations or to depump the atoms negligible while keeping the trigger rate sufficiently high. On the other hand, the large maximal read Rabi frequency $\Omega_{R}^{\max } / 2 \pi \approx 20 \mathrm{MHz}$ ensures that read photons are retrieved 
with a high efficiency. After leaving the cavity, these photons are directed either to a photon correlation or to a homodyne detector, providing complementary information about the measured quantum state. Experimental control is handled by fast digital logic which allows us to acquire their signals only when a trigger photon has been detected during the previous write pulse.

The photon-counting measurement is realized by splitting the photon between two SPCMs 2 and 3 to characterize the single-photon source via the standard second-order intensity correlation function

$$
g^{(2)}(\tau)=\frac{\left\langle\hat{a}^{\dagger}(\tau) \hat{a}^{\dagger}(0) \hat{a}(0) \hat{a}(\tau)\right\rangle}{\left\langle\hat{a}^{\dagger} \hat{a}\right\rangle^{2}}=\frac{\left\langle n_{2}(0) n_{3}(\tau)\right\rangle}{\left\langle n_{2}\right\rangle\left\langle n_{3}\right\rangle},
$$

where $n_{j}$ is the number of photons measured by detector $j$ during a read pulse. Since the data acquisition is triggered by write photons detected at random times, in the expression of the average coincidence rate $\left\langle n_{2}(0) n_{3}(\tau)\right\rangle$, the parameter $\tau$ does not correspond to a physically meaningful time but to a number of trigger events on SPCM 1. The result of this measurement, displayed in Fig. 3, shows that $g^{(2)}(\tau \neq 0) \approx 1$, meaning that the detection events are uncorrelated within different pulses, whereas within the same pulse, $g^{(2)}(0)=0.04 \pm 0.01$, confirming the strongly sub-Poissonian character of the photon source.

In addition to this correlation function, the histogram of photon detection times registered by each detector with a $10 \mathrm{~ns}$ resolution allows us to determine the temporal envelopes of write and read photons. As shown in Fig. 3, the write photon emission profile exactly matches the temporal

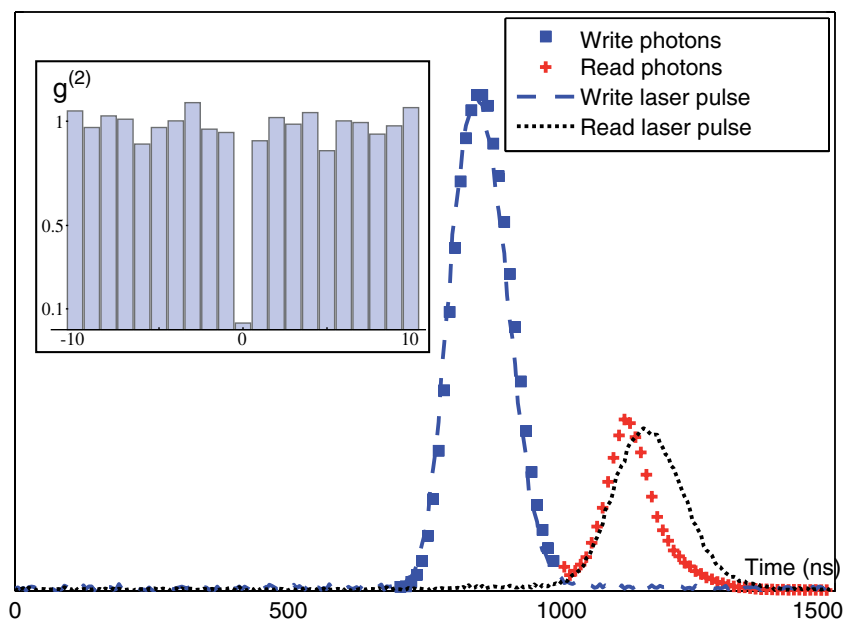

FIG. 3 (color online). Photon-counting measurements. Histograms of arrival times of the write photon on SPCM 1 and of the read photon on SPCM 2 or 3, superimposed with the measured pulse profiles of the write and read pulses (arbitrary vertical units). Inset: Measured correlation function $g^{(2)}$ showing anticorrelation of the photons in a single read pulse down to $g^{(2)}(0) \approx 0.04$ intensity dependence of the write pulse, as expected for a weakly driven spontaneous Raman process. On the other hand, as predicted in [19], the read photon is extracted at the beginning of the read laser pulse and is considerably shorter than the latter, remaining approximately Gaussian with a $1 / e$ half-width of 40 ns.

Finally, knowing the total efficiency $\eta_{c}=37 \pm 1.5 \%$ of the photon counting detection system, we estimate the intrinsic efficiency of our photonic source, defined as the probability to create a free-propagating photon in the analyzed mode each time a trigger write photon was detected, to $\eta=79 \pm 3 \%$. This free-space retrieval efficiency, relevant for most applications, exceeds those observed in previous similar experiments where efficient polariton-tophoton conversions were undermined by outcoupling or filtering losses $[18,20]$.

To analyze the quantum state of the retrieved photon by homodyne tomography, it is sent to interfere on a 50:50 beam splitter with a continuous-wave local oscillator (LO) beam. The lasers producing the read and LO beams are phase locked, which allows us to precisely match the LO and the read photon's frequencies. Approximately $7 \mathrm{~mW}$ of LO power sets the vacuum noise level $\approx 20 \mathrm{~dB}$ above electronic noise. The spatial mode matching between the $\mathrm{LO}$ and the signal is optimized using a probe transmitted through the cavity locked on its fundamental mode. Since our state generation process is phase independent, we do not actively control the phase between the read and the LO beams and assume that the density matrix of the state is diagonal in the Fock basis (if this assumption was wrong, this would only lead to underestimating the state's purity).

Upon detection of a trigger photon, we register $2.2 \mu$ s of homodyne data sampled at a $250 \mathrm{MHz}$ rate. Each data sample $h_{j}(t)$ is then multiplied by a weighting function $f(t)$ and integrated. The obtained value $x_{j}=\int h_{j}(t) f(t)$ corresponds to the measurement of a field's quadrature $\hat{x}$ in the temporal mode defined by $f$. Since the homodyne measurement is sensitive to the field's amplitude, proportional to the square root of its intensity, the optimal weighting function is expected to be a Gaussian $\sqrt{2}$ times wider than the one measured by photon counting. This is confirmed by an independent optimization procedure which yields a $55 \approx$ $40 \times \sqrt{2} \mathrm{~ns} 1 / e$ half-width for the optimal Gaussian weighting function. In order to properly normalize the measured signal, we measure the vacuum noise variance applying to the data the same filtering function $f$ shifted by $T \gg 55 \mathrm{~ns}$, thus selecting a temporally orthogonal mode containing a vacuum state. The measured quadrature probability distribution $P_{0}(x)$ of the vacuum state, shown in Fig. 4, is Gaussian, with a variance normalized to $1 / 2$ by convention. On the other hand, the quadrature distribution $P_{1}(x)$ of the single photon, reconstructed by measuring $\approx 103000$ quadrature values, is strongly non-Gaussian, with a characteristic dip around $x=0$. By applying a maximum likelihood algorithm to this quadrature distribution, 


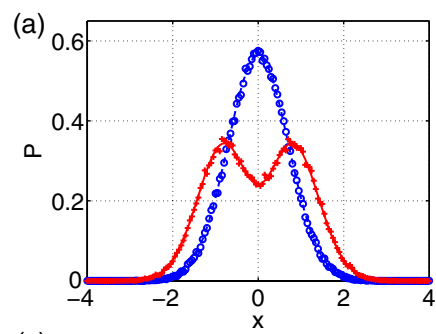

(c)
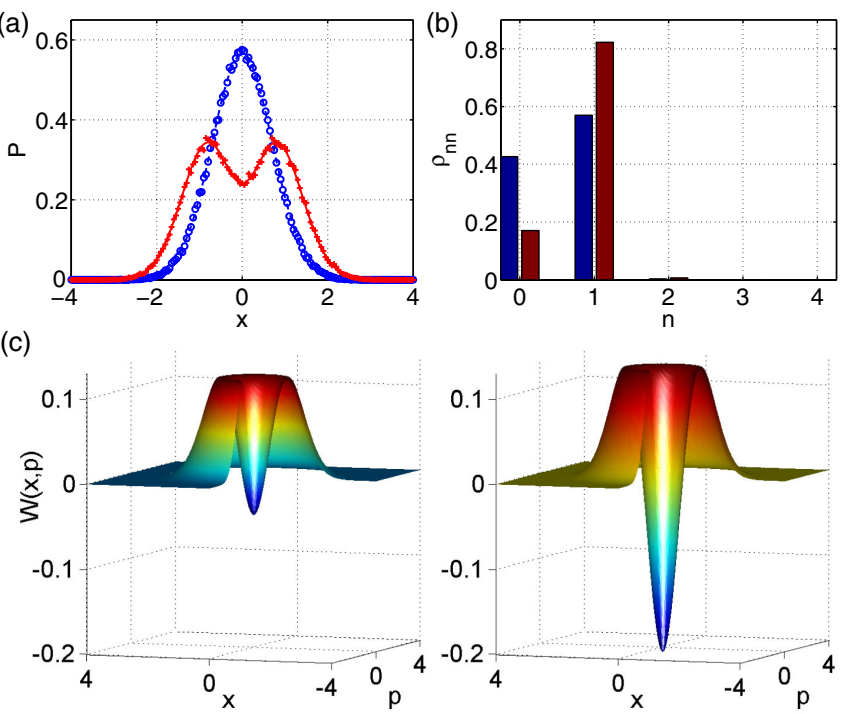

FIG. 4 (color online). Homodyne measurement of the single photon. (a) Measured quadrature probability distribution $P_{0}(x)$ of the vacuum state (blue circles) and $P_{1}(x)$ of the single photon (red crosses), interpolated by the probability distributions estimated by integrating the reconstructed uncorrected Wigner functions along one dimension in phase space. (b) Single-photon density matrix after maximum likelihood reconstruction before (left, blue) and after (right, red) correction for the detector's losses and noise. (c) Corresponding Wigner functions.

we directly obtain the density matrix and the Wigner function of the measured state, displayed in Fig. 4. The density matrix presents a very small two-photon component and a single-photon fraction of $57 \%$. As a result, the Wigner function reconstructed from raw data becomes negative at the origin. The marginal distributions obtained by integrating the vacuum and single-photon Wigner functions overlap very well with the measured quadrature distributions, confirming the consistency of the data analysis process. The fact that the spectral width of the photon, obtained by Fourier transforming its envelope, is narrower than those of the cavity and of the atomic transition (respectively, 2, 10 , and $3 \mathrm{MHz}$ ) shows that the emission process is not spontaneous but coherently driven.

In order to properly characterize the state produced by our source, we can integrate the imperfections of the homodyne detection setup in our maximum likelihood reconstruction procedure. This includes the optical transmission between the vacuum chamber and the detector $\eta_{h d}=82 \pm 1 \%$, the interference visibility between the LO and the signal $\eta_{m}=96.5 \pm 1 \%$, the quantum efficiency of the detector's photodiodes $\eta_{q}=91 \%$, and a $1 \%$ electronic noise contribution. After accounting for these imperfections, the two-photon contribution to the density matrix remains low while the single-photon component increases to $\eta=82 \pm 2 \%$, in excellent agreement with the photoncounting measurement, yielding a strongly nonclassical negative Wigner function.

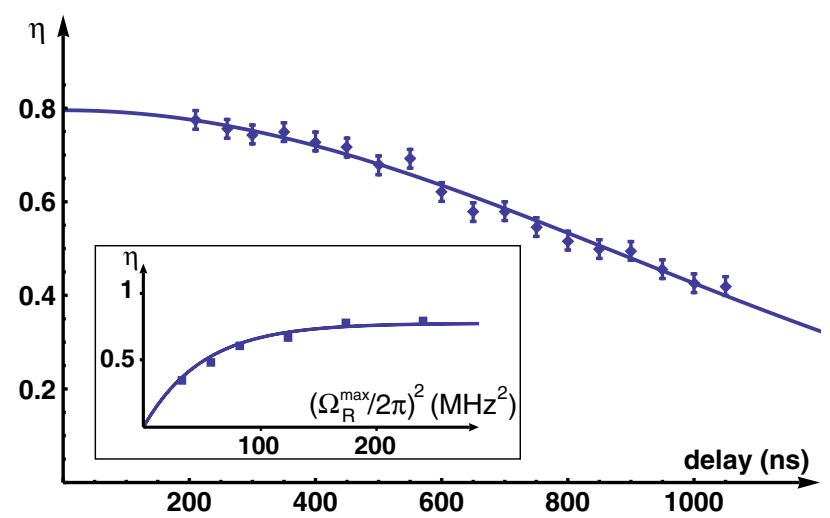

FIG. 5 (color online). Measured single-photon retrieval efficiency $\eta$ as a function of the delay between the write and read pulses, fitted by a Doppler-induced Gaussian decay function with a characteristic time of $\approx 900 \mathrm{~ns}$. Inset: Short-time retrieval efficiency as a function of the squared maximum read Rabi frequency $\left(\Omega_{R}^{\max }\right)^{2}$, superimposed with a realistic model of the experiment (see text).

We have used a theoretical model of the experiment, based on the calculation of [19], incorporating various defects, among which are most importantly the imperfect optical pumping (evaluated to be $92 \%$ ) and the spatial variations of the write-read laser beams and photon modes across the atomic cloud. The maximal retrieval efficiency is consistent with the combination of the limit set by the finite cooperativity with these various imperfections in our setup. The optimal value $C \approx 15$ of the cooperativity, providing the best photon retrieval efficiency given the experimental imperfections, is very similar to the one obtained in previous experiments [18]. The effect of a finite atomic temperature, inducing motional decoherence, can be measured by varying the delay between the write and the read pulses. As shown in Fig. 5, the time dependence of the retrieval efficiency follows the expected Gaussian decay. The measured characteristic time $\tau \approx 900 \mathrm{~ns}$ is in very good agreement with the Doppler time for a Maxwell-Boltzmann distribution of atoms at $T=50 \mu \mathrm{K}\left(\tau_{D}=\sqrt{m / k_{B} T} / 2 k\right.$, where $k$ is the wave vector of $795 \mathrm{~nm}$ light and $m$ is the atomic mass of ${ }^{87} \mathrm{Rb}$ ). This Doppler decay could, in principle, be reduced by placing the atoms in a light shift compensated optical lattice [21] or by increasing the wavelength of the atomic spin wave using an appropriate beam configuration [20], which should not fundamentally impact the purity of the retrieved photon. Our model also accounts for explicit dependence on the read pulse profile and intensity. The agreement with the experiment is satisfactory, as shown, for instance, by the inset in Fig. 5, displaying the short-time retrieval efficiency as a function of the maximal read beam Rabi frequency.

In summary, we experimentally demonstrate a cold atom-based single-photon source that exhibits high modal purity and retrieval efficiency up to $82 \%$. A single excitation can be stored in the cloud with a coherence time of 
$900 \mathrm{~ns}$ and retrieved on demand as a single photon in a very well defined quantum state, consistently characterized by photon counting and homodyne tomography, revealing nonclassical features in both the intensity and the light field. Since homodyne detectors can measure phase-dependent states with arbitrary photon numbers, this demonstrates that homodyne analysis methods can be conveniently applied to more complex state production schemes available in the cold atom ensemble systems, in particular, those based on few-photon nonlinearities induced by atomic interactions.

This work was supported by the EU through the ERC Advanced Grant 246669 "DELPHI" and the Collaborative Project 600645 "SIQS." We thank MarcHenri Pottier for assistance with the experimental control software.

*erwan.bimbard@institutoptique.fr

†Present address: Laboratoire Kastler Brossel, Université Pierre et Marie Curie, École Normale Supérieure, CNRS, 4 place Jussieu, 75252 Paris Cedex 05, France.

[1] A. I. Lvovsky, B. C. Sanders, and W. Tittel, Nat. Photonics 3, 706 (2009).

[2] Y. O. Dudin and A. Kuzmich, Science 336, 887 (2012).

[3] T. Peyronel, O. Firstenberg, Q.-Y. Liang, S. Hofferberth, A. V. Gorshkov, T. Pohl, M. D. Lukin, and V. Vuletić, Nature (London) 488, 57 (2012).

[4] D. Maxwell, D. J. Szwer, D. Paredes-Barato, H. Busche, J. D. Pritchard, A. Gauguet, K. J. Weatherill, M. P. A. Jones, and C. S. Adams, Phys. Rev. Lett. 110, 103001 (2013).

[5] J. K. Thompson, J. Simon, H. Loh, and V. Vuletić, Science 313, 74 (2006).

[6] D. Felinto, C. W. Chou, J. Laurat, E. W. Schomburg, H. de Riedmatten, and H. J. Kimble, Nat. Phys. 2, 844 (2006).
[7] T.Chanelière, D. N. Matsukevich, S. D. Jenkins, S.-Y. Lan, R. Zhao, T. A. B. Kennedy, and A. Kuzmich, Phys. Rev. Lett. 98, 113602 (2007).

[8] Z.-S. Yuan, Y.-A. Chen, S. Chen, B. Zhao, M. Koch, T. Strassel, Y. Zhao, G.-J. Zhu, J. Schmiedmayer, and J.-W. Pan, Phys. Rev. Lett. 98, 180503 (2007).

[9] A. I. Lvovsky and M. G. Raymer, Rev. Mod. Phys. 81, 299 (2009).

[10] K. Honda, D. Akamatsu, M. Arikawa, Y. Yokoi, K. Akiba, S. Nagatsuka, T. Tanimura, A. Furusawa, and M. Kozuma, Phys. Rev. Lett. 100, 093601 (2008).

[11] J. Appel, E. Figueroa, D. Korystov, M. Lobino, and A. I. Lvovsky, Phys. Rev. Lett. 100, 093602 (2008).

[12] J. Cviklinski, J. Ortalo, J. Laurat, A. Bramati, M. Pinard, and E. Giacobino, Phys. Rev. Lett. 101, 133601 (2008).

[13] K. Jensen, W. Wasilewski, H. Krauter, T. Fernholz, B. M. Nielsen, M. Owari, M. B. Plenio, A. Serafini, M. Wolf, and E. S. Polzik, Nat. Phys. 7, 13 (2011).

[14] M. Hosseini, G. Campbell, B. M. Sparkes, P. K. Lam, and B. C. Buchler, Nat. Phys. 7, 794 (2011).

[15] A. MacRae, T. Brannan, R. Achal, and A. I. Lvovsky, Phys. Rev. Lett. 109, 033601 (2012).

[16] J.-I. Yoshikawa, K. Makino, S. Kurata, P. van Loock, and A. Furusawa, Phys. Rev. X 3, 041028 (2013).

[17] L.-M. Duan, M. D. Lukin, J. I. Cirac, and P. Zoller, Nature (London) 414, 413 (2001).

[18] J. Simon, H. Tanji, J. K. Thompson, and V. Vuletić, Phys. Rev. Lett. 98, 183601 (2007).

[19] J. Stanojevic, V. Parigi, E. Bimbard, R. Tualle-Brouri, A. Ourjoumtsev, and P. Grangier, Phys. Rev. A 84, 053830 (2011).

[20] X.-H. Bao, A. Reingruber, P. Dietrich, J. Rui, A. Duck, T. Strassel, L. Li, N.-L. Liu, B. Zhao, and J.-W. Pan, Nat. Phys. 8, 517 (2012).

[21] A. G. Radnaev, Y. O. Dudin, R. Zhao, H. H. Jen, S. D. Jenkins, A. Kuzmich and T. A. B. Kennedy, Nat. Phys. 6, 894 (2010). 